Meta

Journal des traducteurs

Translators' Journal

\title{
L'accréditation des interprètes judiciaires au Palais de justice de Montréal
}

\author{
Christine Viens, Georges L. Bastin, Solange Duhamel et Roselyne Moreau
}

Volume 47, numéro 2, juin 2002

Traduction et terminologie juridiques

URI : https://id.erudit.org/iderudit/008016ar

DOI : https://doi.org/10.7202/008016ar

Aller au sommaire du numéro

Éditeur(s)

Les Presses de l'Université de Montréal

ISSN

0026-0452 (imprimé)

1492-1421 (numérique)

Découvrir la revue

Citer cet article

Viens, C., Bastin, G. L., Duhamel, S. \& Moreau, R. (2002). L’accréditation des interprètes judiciaires au Palais de justice de Montréal. Meta, 47(2), 289-293.

https://doi.org/10.7202/008016ar d'utilisation que vous pouvez consulter en ligne. 


\title{
L'accréditation des interprètes judiciaires au Palais de justice de Montréal
}

\author{
CHRISTINE VIENS, GEORGES L. BASTIN, \\ SOLANGE DUHAMEL ET ROSELYNE MOREAU
}

La Charte canadienne des droits et libertés reconnaît le droit aux services d'un interprète, en matière criminelle et pénale, à toute partie ou au témoin qui ne peut suivre les procédures, soit parce qu'il ne comprend pas ou ne parle pas la langue employée, soit parce qu'il est atteint de surdité. Ce droit existe quelle que soit la langue. Le droit à l'assistance d'un interprète est également reconnu par la Charte québécoise à tout accusé.

La complexité de la profession d'interprète est évidente dans le milieu judiciaire, où le défaut de rendre un service de qualité peut avoir un impact majeur sur les droits et libertés des personnes. Les enjeux sont importants; la violation des droits de l'accusé peut faire avorter un procès.

Nous dresserons d'abord un portrait de nos services, puis, de la démarche qui a mené tout récemment à l'adoption d'un processus de qualification des interprètes judiciaires appelés à agir pour le ministère de la justice du Québec. Enfin, nous décrirons les principaux éléments de ce processus.

\section{A. Le Service d'interprétation et de traduction judiciaires}

Le ministère de la Justice fournit gratuitement des services professionnels d'interprètes et de traducteurs, conformément aux Chartes, aux parties, témoins ou accusés, devant les Cours de justice de juridictions criminelle et pénale.

Ces services sont également fournis sans frais aux personnes atteintes de surdité, muettes ou malentendantes devant toutes les Cours, y compris les Cours de juridiction civile et pour les entrevues avec certains services du palais.

Enfin, le ministère défraie les services d'interprètes en langues autochtones pour les bénéficiaires de la Convention de la Baie-James et du Nord québécois, ainsi que pour ceux de la Convention du Nord-Est québécois.

\section{a) Responsabilité}

L'accréditation des interprètes, sauf en langues autochtones et pour les sourds-muets et malentendants, est centralisée à Montréal.

\section{b) Statistiques}

Au cours de l'exercice financier 2000-2001, plus de 200 interprètes-traducteurs (accrédités ou non) ont été engagés par le ministère de la Justice pour une dépense totalisant près d'un million de dollars (930 400\$) en honoraires, frais de séjour et de déplacement. 


\section{c) Volume d'activités}

Le volume en demandes d'interprètes varie d'année en année et de région en région, mais il est de beaucoup plus élevé à Montréal qu'ailleurs.

La population de Montréal vient de toutes les parties du monde et les demandes suivent en conséquence. Le visage de Montréal est de plus en plus multiethnique.

\section{d) Langues d'interprétation}

Actuellement, nous fournissons des interprètes dans plus de 60 langues et dialectes.

Les vagues d'immigration affectent nos services. Un grand nombre de nouveaux arrivants issus d'une immigration massive (par ex.: des réfugiés) ne parlent pas les langues officielles du Canada. Ils ont donc besoin d'interprètes, du moins à leur arrivée, jusqu'au moment où ils apprennent l'une ou l'autre de nos langues officielles reconnues à la Cour, le français ou l'anglais.

L'actualité judiciaire, les tensions interraciales et les divers événements qui peuvent, à certains moments, toucher des individus de certaines communautés ethniques ont aussi un impact sur la demande. C'est donc par vagues que l'on reçoit des demandes en certaines langues.

Il y a 4 ans, par exemple, la langue tamoule était très en demande. Deux bandes rivales se livraient bataille; les agresseurs d'un jour étant les victimes du lendemain. L'année 2000 a été celle du punjabi et de l'ourdou, ainsi que du vietnamien et du roumain. Certaines langues ne sont à peu près pas demandées pendant un certain temps et puis, pendant quelques mois, les interprètes qui travaillent dans ces langues sont très sollicités. Ce fut le cas, l'an dernier, pour l'allemand, le hongrois, l'arménien et l'albanais. Il y a plusieurs années, l'arabe, l'espagnol et l'italien étaient des langues très en demande. Aujourd'hui, le besoin est toujours présent mais la demande a beaucoup diminué.

Comme on peut l'imaginer, tous ces éléments rendent aléatoire la planification du recrutement des interprètes et peuvent parfois causer des maux de tête à la coordonnatrice. Toujours dans l'obligation de fournir un service à la Cour, cette dernière est parfois confrontée à la rareté des ressources dans une langue alors que la demande est soudaine. C'est dans ces moments que la collaboration entre les différents services d'interprétation, comme ceux de l'immigration, est particulièrement appréciée.

\section{e) L'assignation des interprètes}

L'assignation des interprètes se fait dans le respect des règles d'équité dans l'octroi des contrats. La coordonnatrice assigne par ailleurs les interprètes devant la Cour en fonction des langues et de la complexité des causes.

\section{f) Qualités requises d'un interprète judiciaire et normes d'interprétation}

Les qualités requises d'un interprète judiciaire peuvent être résumées comme suit:

- les interprètes judiciaires doivent être souples, impartiaux, discrets, avoir une excellente mémoire et être capables de rendre fidèlement les audiences à la Cour. (cf. «Guide», page 9); 
- dans les Cours de justice, on peut voir dans la même cause un interprète faire de l'interprétation simultanée et de l'interprétation consécutive;

- pour donner une bonne prestation, il est très important que l'interprète connaisse le processus judiciaire et la terminologie juridique.

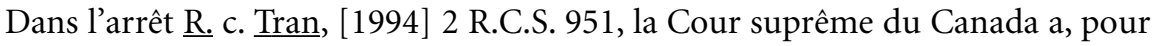
la première fois, établi, en 1994, une norme d'interprétation garantie fondée sur cinq critères:

- la continuité

L'accusé a droit à l'assistance d'un interprète pendant toutes les procédures c'est-à-dire sans pause ni interruption (sans qu'il ne s'agisse nécessairement de la même personne);

- la fidélité

L'interprétation doit, autant que possible, reprendre chaque mot et chaque idée, car les résumés seraient insatisfaisants;

- l'impartialité

L'interprète doit être objectif et impartial et, autant que possible, ne doit être lié à aucune des parties au litige;

- la compétence

L'accusé a droit à un interprète dont la compétence est reconnue par le tribunal;

- la concomitance

L'interprétation doit être une interprétation consécutive ou simultanée afin de permettre à l'accusé de réagir au moment opportun.

\section{B. Le processus de qualification des interprètes}

Tout ce qui précède met en évidence la nécessité de s'assurer de la compétence des interprètes référés à la Cour. Le juge doit évaluer le besoin de recourir à un interprète (lorsque les intérêts vitaux de l'accusé sont en jeu); de plus, il s'assure du respect des cinq critères établis par la Cour suprême. Mais, en pratique, il revient au ministère de la Justice de voir au recrutement, à la qualification et à l'assignation des interprètes.

Actuellement, quelque 160 interprètes contractuels sont accrédités par le ministère, c'est-à-dire sont officiellement reconnus aptes à agir devant les Cours de justice. Ces interprètes sont inscrits dans un fichier, selon la langue, la formation et l'expérience. Ils signent un contrat d'un an avec le ministère de la Justice, contrat renouvelé annuellement. À défaut d'interprètes accrédités disponibles dans une langue donnée, un interprète non accrédité peut être engagé, sous réserve de son acceptation par le tribunal. Il signe alors un contrat de courte durée qui peut être renouvelé selon les besoins.

Au printemps 1999, un comité a été formé pour assurer la mise en œuvre du rapport d'un groupe de travail chargé de dégager des orientations sur l'accréditation des interprètes. Ce comité était composé de membres de la Direction de services judiciaires pénaux à Montréal $^{1}$ et a compté sur la collaboration de l'OTTIAQ et de l'Université de Montréal' ${ }^{2}$ M ${ }^{\mathrm{e}}$ André Ménard et madame Denise Basque ont également apporté leur soutien.

La démarche menant à l'élaboration du processus de qualification des interprètes judiciaires a été laborieuse, notamment en raison de la complexité et de la spécialisation du domaine, de la nécessité d'explorer plusieurs avenues avant d'arrêter une 
orientation et de la disponibilité limitée des intervenants qui se sont penchés sur la question.

La qualification comprend une entrevue, un test de connaissance et un test d'aptitude à l'interprétation.

Au cours de ses travaux, le comité a consulté plusieurs provinces et plus particulièrement apprécié le processus d'accréditation en Ontario, dont il s'est, en partie, inspiré surtout pour sa valeur pédagogique. Ainsi, le comité a obtenu l'autorisation de traduire et d'adapter le «Freelance Court Interpreter Handbook» réalisé en 1995 par le ministère du Procureur général de l'Ontario.

Après avoir mis à jour ce document sur le plan juridique, il a été traduit et adapté à la situation québécoise pour produire en janvier 2001 le «Guide à l'intention des interprètes judiciaires » en versions française et anglaise.

Le «Guide à l'intention des interprètes judiciaires» comprend, tout d'abord, les règles de conduite professionnelle et ensuite, sept chapitres portant respectivement sur les techniques d'interprétation judiciaires, les règles de conduite devant les Cours de justice, une description sommaire du système judiciaire québécois, des notions de base sur la procédure criminelle et sur les infractions les plus courantes, ainsi qu'un survol en matière de droit de la jeunesse et des petites créances. À la fin de chaque chapitre, on trouve une série de questions qui permettent au candidat de vérifier son niveau d'assimilation de la matière.

\section{a) Test de connaissances}

Ce guide sert de base au test de connaissances. Le test de connaissances est un examen écrit comprenant 10 questions choisies au hasard parmi les quelque 50 questions contenues au guide. Le candidat-interprète doit répondre correctement à 6 questions sur 10. Ce test est éliminatoire.

\section{b) Test d'aptitude à l'interprétation judiciaire}

Quant au test d'aptitude à l'interprétation judiciaire, il consiste en un enregistrement audiovisuel en trois parties qui permet d'évaluer la mémoire du candidat ainsi que ses habiletés en interprétation consécutive et simultanée. Les tests sont produits, pour le moment, en français et en anglais seulement.

Le candidat peut se servir d'écouteurs pour écouter la cassette audiovisuelle lors de l'examen. La performance du candidat est enregistrée sur cassette audio seulement. La note de passage est de 3 sur 5 et ce test est éliminatoire.

L'enregistrement des tests d'aptitude à l'interprétation judiciaire sur un support audiovisuel est une nouveauté, les tests étant auparavant enregistrés sur cassette audio seulement. Les enregistrements se sont déroulés dans une salle de Cour avec des comédiens amateurs dont faisaient partie certains membres du comité. Sur chaque cassette, les timbres de voix et les accents sont donc des plus variés. Cette façon de faire permet de reproduire, autant que possible, des conditions se rapprochant de la réalité vécue au tribunal.

Le coordonnateur provincial est responsable de la qualification et, le cas échéant, du classement de l'interprète. Il veille à la correction des tests de la façon suivante: 
- Le test de connaissances est corrigé par une personne membre du Barreau du Québec, notamment le conseiller juridique de la Direction des services judiciaires de la Métropole ou un membre de son équipe.

- Le test d'aptitude à l'interprétation judiciaire est corrigé par un interprète chevronné non-inscrit sur la liste des interprètes qualifiés pour le palais de justice concerné par la candidature. Ce dernier remplit la grille d'évaluation en tenant compte des critères suivants: l'intelligibilité, l'information et la présentation.

Pour obtenir la qualification, le candidat doit passer avec succès le test de connaissances et le test d'aptitude.

Le candidat qui échoue les deux tests ou l'un de ces tests peut se reprendre après un délai de carence de 6 mois de la date de l'examen. Dans le cas où le candidat n'a réussi qu'un seul test, les résultats positifs de ce test sont valables pour un an.

\section{c) Entrevue}

Avant de procéder aux tests, le coordonnateur régional fixe une rencontre avec le candidat afin de lui expliquer les conditions de travail de l'interprète judiciaire, de l'informer des modalités du classement et des tarifs applicables. Cette rencontre permet au coordonnateur régional d'évaluer l'intérêt, la motivation et le comportement du candidat.

C'est également le coordonnateur régional qui veille à la formation de l'interprète nouvellement qualifié en tenant compte de ses besoins et de son expérience déjà acquise devant les cours de justice. La formation porte entre autres sur les matières contenues dans le «Guide à l'intention des interprètes judiciaires». Il peut s'agir aussi d'observation en salle d'audience et de sessions d'interprétation parrainées par un interprète d'expérience servant de mentor.

\section{Conclusion}

Il est prématuré de tirer des conclusions de ce nouveau système d'accréditation. Deux tests ont été administrés avec succès au cours du premier trimestre 2001 et une vingtaine le seront dans le courant de l'année.

\section{NOTES}

1. Solange Duhamel, Roselyne Moreau et Me Christine Viens.

2. Georges Aubé, conseiller juridique à l'OTTIAQ (Office des traducteurs, terminologues et interprètes agrées du Québec) et le professeur Georges L. Bastin, coordonnateur du programme de maîtrise en interprétation à l'Université de Montréal. 